\title{
Mucosa-Associated Escherichia coli in Colorectal Cancer Patients and Control Subjects: Variations in the Prevalence and Attributing Features
}

\author{
Roghayeh Nouri, ${ }^{1,2}$ Alka Hasani ${ }^{\mathbb{D}},{ }^{2,3}$ Kourosh Masnadi Shirazi, \\ Mohammad Reza Alivand $\mathbb{D},{ }^{5}$ Bita Sepehri, ${ }^{4}$ Simin Sotoudeh, ${ }^{4}$ Fatemeh Hemmati, ${ }^{1,2}$ \\ Afshin Fattahzadeh, ${ }^{4}$ Babak Abdinia $\mathbb{D}^{6}{ }^{6}$ and Mohammad Ahangarzadeh Rezaee $\mathbb{i D}^{2,3,7}$ \\ ${ }^{1}$ Student Research Committee, Tabriz University of Medical Sciences, Tabriz, Iran \\ ${ }^{2}$ Infectious and Tropical Diseases Research Center, Tabriz University of Medical Sciences, Tabriz, Iran \\ ${ }^{3}$ Department of Medical Microbiology, Faculty of Medicine, Tabriz University of Medical Sciences, Tabriz, Iran \\ ${ }^{4}$ Liver and Gastrointestinal Diseases Research Center, Tabriz University of Medical Sciences, Tabriz, Iran \\ ${ }^{5}$ Department of Medical Genetics, Faculty of Medicine, Tabriz University of Medical Sciences, Tabriz, Iran \\ ${ }^{6}$ Pediatric Health Research Center, Tabriz University of Medical Sciences, Tabriz, Iran \\ ${ }^{7}$ Clinical Research Development Unit of Children Educational, Research and Treatment Center, \\ Tabriz University of Medical Sciences, Tabriz, Iran
}

Correspondence should be addressed to Mohammad Ahangarzadeh Rezaee; rezaee@tbzmed.ac.ir

Received 1 August 2021; Revised 30 September 2021; Accepted 26 October 2021; Published 9 November 2021

Academic Editor: Tingtao Chen

Copyright (C) 2021 Roghayeh Nouri et al. This is an open access article distributed under the Creative Commons Attribution License, which permits unrestricted use, distribution, and reproduction in any medium, provided the original work is properly cited.

\begin{abstract}
Accumulating evidence indicates that specific strains of mucosa-associated Escherichia coli (E. coli) can influence the development of colorectal carcinoma. This study aimed to investigate the prevalence and characterization of mucosa-associated $E$. coli obtained from the colorectal cancer (CRC) patients and control group. At two referral university-affiliated hospitals in northwest Iran, 100 patients, 50 with CRC and 50 without, were studied over the course of a year. Fresh biopsy specimens were used to identify mucosa-associated E. coli isolates after dithiothreitol mucolysis. To classify the E. coli strains, ten colonies per sample were typed using enterobacterial repetitive intergenic consensus-based PCR (ERIC-PCR). The strains were classified into phylogroups using the quadruplex PCR method. The PCR method was used to examine for the presence of cyclomodulin, bfp, st $x 1$, stx2, and eaeencoding genes. The strains were tested for biofilm formation using the microtiter plate assay. CRC patients had more mucosaassociated E. coli than the control group $(p<0.05)$. Enteropathogenic Escherichia coli (EPEC) was also found in $23 \%$ of CRC strains and $7.1 \%$ of control strains $(p<0.05)$. Phylogroup A was predominant in control group specimens, while $E$. coli isolates from CRC patients belonged most frequently to phylogroups D and B2. Furthermore, the frequency of cyclomodulin-encoding genes in the CRC patients was significantly higher than the control group. Around $36.9 \%$ of E. coli strains from CRC samples were able to form biofilms, compared to $16.6 \% \mathrm{E}$. coli strains from the control group $(p<0.05)$. Noticeably, cyclomodulin-positive strains were more likely to form biofilm in comparison to cyclomodulin-negative strains $(p<0.05)$. In conclusion, mucosaassociated E. coli especially cyclomodulin-positive isolates from B2 and D phylogroups possessing biofilm-producing capacity colonize the gut mucosa of CRC patients.
\end{abstract}

\section{Introduction}

Colorectal cancer (CRC) is the world's third most common cancer and the second leading cause of cancer death [1].
Because of its high morbidity and mortality rate, CRC is an important public health issue [2]. Mutations that occur in tumor suppressor genes, oncogenes, and genes related to DNA repair mechanisms can lead to CRC. Depending on the 
origin of the mutations, colorectal carcinomas are divided into three types: sporadic (70\%), familial (25\%), and inherited (5\%) [3].

The human gut microbiota contains over more than 1,000 microbial species, adding together $10^{14}$ microorganisms [4] that play an essential role in many important physiological processes, such as food digestion, metabolism, and immune response [5]. Shifts in the composition of these resident microbiota, the so called microbial dysbiosis, have been found to cause various diseases, including $\mathrm{CRC}[6,7]$, cardiovascular disease, inflammatory bowel disease, and diabetes mellitus [6]. Approximately $20 \%$ of all cancers in humans can be related to infectious agents. Gut bacteria may be involved in the initiation or progression of sporadic CRC via a variety of mechanisms, including inducing inflammation, generating reactive oxygen species, and producing genotoxins [8]. Published literature suggests that some bacterial species such as Bacteroides fragilis [9], Streptococcus bovis, Fusobacterium spp., Helicobacter pylori, and Clostridium septicum are associated with colorectal carcinogenesis [10]. Moreover, considering the fact that E. coli is the most common facultative anaerobic resident in human gut flora, several research studies have shown a strong link between mucosa-associated E. coli and CRC [8, 11-13]. Moreover, E. coli strains are categorized into phylogenetic groups (A, B1, B2, C, D, E, and F) based on the existence or absence of a variety of genes and DNA fragment [14]. Pathogenic E. coli strains are mostly found in the B2 or D phylogroups, while commensal strains are mostly found in groups A and B1 [15].

Interestingly, enteropathogenic Escherichia coli (EPEC) is commonly found in CRC patients, in contrast to their occasional presence in the control subjects [16]. Additionally, E. coli isolates that carry the eae gene (encoding for intimin protein) are able to attach the intestinal epithelium and are classed as attaching and effacing E. coli (AEEC). More noteworthy, EPEC is also the most widely found AEEC bacteria in humans. This bacterium suppresses the expression of key DNA mismatch repair proteins (MMR), which suggests that chronic mucosal EPEC infection can contribute to the development of CRC tumors [17]. Typical EPEC strains (tEPEC) have the eae and $b f p$ (gene encoding for bundle-forming pili) genes but no Shiga toxin genes (stx). Often known as atypical EPEC, certain clinical isolates of EPEC lack the $b f p$ gene [18].

Usually, pathogenic E. coli strains produce several toxins called cyclomodulins including the cytotoxic necrotizing factor (CNF), cycle inhibiting factor (Cif), colibactin, and cytolethal distending toxins (CDTs). The colibactin is a toxin produced by polyketide synthetase ( $p k s)$ [8]. Cyclomodulins are attracting growing attention due to their ability to influence cellular differentiation, apoptosis, and cell proliferation by disrupting the eukaryotic cell cycle and/or promoting DNA damage [19]. For example, B2 E. coli strains carrying the cyclomodulin-encoding genes are more prevalent in colon tumor biopsies in comparison to control samples suggesting a possible role of these isolates in CRC carcinogenesis $[12,20]$.

In addition, recent studies have shown that bacterial biofilms are associated with human colon cancer [21, 22].
Mucus-invasive bacterial biofilms, for example, were found on the colon mucosa of CRC patients more frequently than in healthy subjects [23]. Biofilm formation causes an increase in epithelial junction permeability in both normal and cancerous colons, which can facilitate direct access to mutagens from bacteria to the colonic epithelial surface and encourage procarcinogenic tissue inflammation [24]. In general, E. coli appears to play a significant role in CRC carcinogenesis, but there are little details on the characterization of colonic mucosa-associated E. coli from CRC patients. Therefore, the aim of this analysis was to compare the presence and characteristics of mucosa-associated E. coli isolates in biopsy specimens from CRC patients and a control group.

\section{Materials and Methods}

2.1. Patients. Between June 2019 and June 2020, 100 patients were examined at two referral university-affiliated hospitals in northwest Iran (Table 1). Among these, 50 patients had CRC, while equal number of patients without CRC had piles $(n=26)$, pruritus ani $(n=12)$, irritable bowel syndrome (IBS) $(n=2)$, and no symptoms (refer for screening; $n=10$ ) were taken as controls into the study.

Clinical features (such as weight loss, rectal bleeding, abdominal pain, and bowel habit changes), a positive fecal occult blood test, colonoscopy, and pathology findings were used to diagnose CRC. Furthermore, IBS was diagnosed based on the presence of clinical indications and symptoms (Rome criteria).

In this study, noncancerous individuals without acute or chronic inflammation were included in the control group. In addition, inclusion criteria for CRC patients were a histological diagnosis of malignant tumor without any previous treatment. Moreover, patients who had taken antibiotics in the four weeks leading up to the endoscopy were also excluded from the study.

2.2. Sample Treatment. During colonoscopy, biopsy specimens were obtained from the colon and rectum using regular endoscopic forceps. The neoplastic characteristics of the biopsy samples were verified by pathological results. The mucosal biopsy specimens were placed in an Eppendorf tube containing normal saline and transferred to the lab [16]. The samples were washed three times in $5 \mathrm{~mL}$ PBS to remove the fecal bacteria [12]. Then, in order to remove the mucus layer, fresh biopsy specimens were transferred to a microtube containing $500 \mu \mathrm{L}$ of $0.016 \% \mathrm{wt} / \mathrm{vol}$ dithiothreitol solution and rotated for 10 minutes [25]. The obtained supernatant was inoculated in nutrient broth (NB) and incubated at $37^{\circ} \mathrm{C}$ for 24 hours. After the specified period, broth culture was subcultured on MacConkey agar. E. coli isolates were identified by standard conventional biochemical tests.

\subsection{ERIC-PCR Typing and Phylogenetic Classification.}

Ten colonies of $E$. coli from each positive sample were typed with enterobacterial repetitive intergenic consensus-based PCR (ERIC-PCR) as described by Versalovic et al. (1991) 
TABLE 1: Characteristics of CRC patients and control group subjects.

\begin{tabular}{lccc}
\hline Characteristics & CRC patients $(n=50)$ & Control group $(n=50)$ & $P$ value \\
\hline Age, median $($ range $)$ & $64(40-83)$ & $56(31-82)$ & $P>0.05$ \\
Gender, male/female & $27 / 23$ & $17 / 33$ & $P>0.05$ \\
BMI $\left(\mathrm{kg} / \mathrm{m}^{2}\right)($ mean $\pm \mathrm{SD})$ & $25.6 \pm 3.1$ & $26.1 \pm 3.3$ & $P>0.05$ \\
\hline
\end{tabular}

[26]. All E. coli strains were then divided to phylogroups based on the presence of the DNA fragment (TSPE4.C2) and $\operatorname{arp} A, \operatorname{chu} A, \operatorname{trp} A$, and $y j a A$ genes, which were detected by the quadruplex PCR method proposed by Clermont et al. [14].

2.4. Detection of Cyclomodulin Genes and Other Virulence Factors. The PCR method was performed using primers specific for the cyclomodulin (cif, cnf1, cnf2, cnf3, cdtB-I, $c d t B-I I, c d t B-I I I, c d t B-I V, c d t B-V$, and $p k s$ genomic island), $c l b A, c l b Q$ [27], $b f p, s t x 1, s t x 2$, and intimin (eae)-encoding genes [25].

The template DNA from $E$. coli isolates was extracted using the boiling water method, as described previously [27]. The PCR reaction was carried out in a $50 \mu \mathrm{L}$ mixture containing $10-100 \mathrm{ng}$ of the template DNA, $0.2 \mathrm{mM}$ each dNTPs, $0.5 \mathrm{pmol}$ of each primer, $3 \mathrm{mM} \mathrm{MgCl}_{2}$, and $1.0 \mathrm{U}$ of DNA polymerase (Yekta Tajhiz Azma, Iran), in the corresponding reaction buffer.

2.5. Biofilm Production Assay. Biofilm formation assays were carried out by the microtiter plate method, as described before using $2 \%$ crystal violet. Optical density (OD) of each stained well was measured at $570 \mathrm{~nm}$. The cutoff OD was defined as three standard deviations above the mean OD of the negative control. TSB medium without bacteria was considered as a negative reagent control. All isolates were classified into no or weak biofilm producers, moderate biofilm producers, or high biofilm producers [28].

2.6. Statistical Analysis. In this study, the results were analyzed by the chi-square test or Fisher's exact test using SPSS software for Windows (version 23 SPSS Inc., Chicago, IL, USA). A $p$ value less than 0.05 was considered a statistically significant difference.

\section{Results}

3.1. E. coli Strains in CRC and Control Group Specimens. The investigation of the presence of $E$. coli isolates showed that the number of specimen without $E$. coli was remarkably higher in control specimens $(20 \%, n=10 / 50)$ than in those with CRC patients $(4 \%, n=2 / 50), P=0.028$. In this study, some specimens carried more than one $E$. coli strains. As some specimens carried more than one E. coli strains, a total of $65 \mathrm{E}$. coli strains from CRC patients and 42 E. coli strains from the control group were taken into the study (Figure 1).
3.2. Prevalence of EPEC in CRC and Control Group Samples. EPEC are defined as isolates that have the eae and $b f p$ genes but no Shiga toxin genes. Often known as atypical EPEC, certain clinical isolates of EPEC lack the $b f p$ gene.

The eae gene was detected in $23 \%$ E. coli isolated from clinical specimen obtained from the CRC group, but only in $7.1 \%$ E. coli isolated from the control group, the difference being statistically significant $(p=0.036)$. Moreover, all eaepositive strains were negative for stx 1 and stx2 genes. Interestingly, all EPEC strains were atypical EPEC (PCR negative for bfp) (Figure 2).

3.3. Distribution of E. coli Phylogroups according to the Specimen Origin. Phylogenetic studies revealed that the prevalence of phylogroups in E. coli strains from CRC specimens and controls varied significantly. As given in Table 2, phylogroup D was the predominant type in E. coli obtained from CRC patients (41.5\%), followed by phylogroups B2 (36.9\%), A (13.9\%), and B1 (7.7\%). On the other hand, E. coli isolated in the control group most frequently belonged to phylogroup A (38.1\%), followed by phylogroups B1 (19\%), D (16.7\%), B2 (11.9\%), E (9.5\%), and F (4.7\%).

3.4. Distribution of Cyclomodulin-Encoding Genes according to the Sample Origin and Phylogroups. As given in Table 3, the increased frequencies of cyclomodulin-encoding genes on CRC samples $(30.7 \%, n=20 / 65)$ compared to control tissues were statistically significant $(4.7 \%, n=2 / 42)$, $p=0.001$. It is worth noting that the cif gene was the only gene revealed $(4.7 \%)$ among cyclomodulin-encoding genes in E. coli strains obtained from the control group. Among E. coli strains from CRC patients, the strains harboring cif, cnfl, and pks genes represented $16.9 \%, 12.3 \%$, and $9.2 \%$ of the total strains isolated, respectively. Additionally, $7.7 \%$ $(n=5)$ E. coli strains from CRC patients carried more than one cyclomodulin-encoding genes. Moreover, cnf2, cnf3, and $c d t B$ genes were not found in any of the strains from CRC specimens.

All strains of patients in the CRC group harboring $p k s$ genomic island belonged to phylogroup B2. Moreover, 50\% E. coli strains in this group harboring $c n f 1$ gene belonged to phylogroup B2, while $50 \%$ E. coli strains belonged to phylogroup D. The distribution of phylogroups amongst E. coli strains of the CRC group possessing cif gene was as follows: $54.5 \%$ belonged to phylogroup $\mathrm{D}$, followed by phylogroups A (18.2\%), B2 (18.2\%), and B1 (9.1\%) (Table 3).

3.5. Biofilm-Forming Ability of E. coli Strains from the Control Group and CRC Specimens. As given in Table 4, in this study, there was a significant difference between the ability to form 


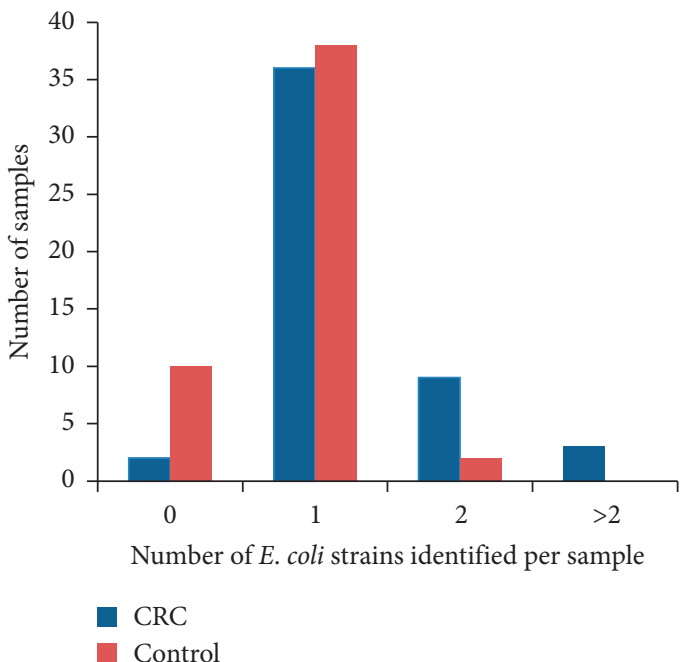

(a)

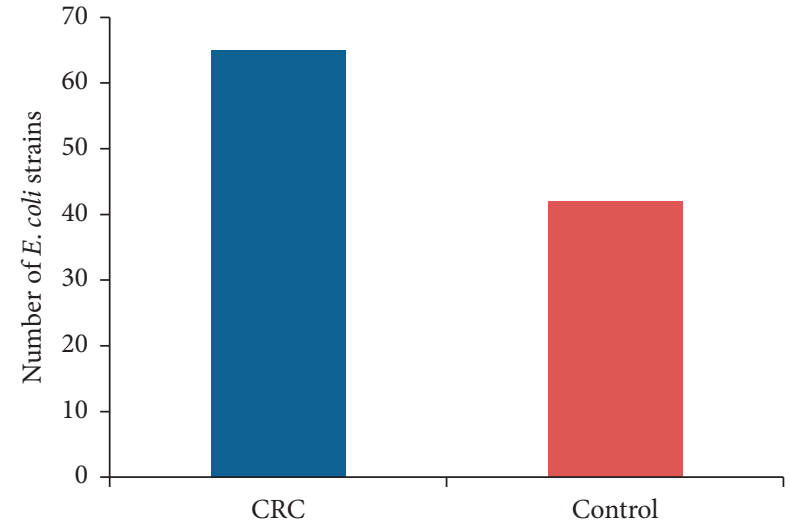

(b)

Figure 1: Number of E. coli strains obtained from 100 subjects comprising 50 CRC patients and the equivalent number of control subjects. (a) Some specimens did not carry any strain of E. coli and some specimens carried one or more than one E. coli strains. (b) A total of 65 E. coli strains from CRC patients and $42 \mathrm{E}$. coli strains from the control group identified.

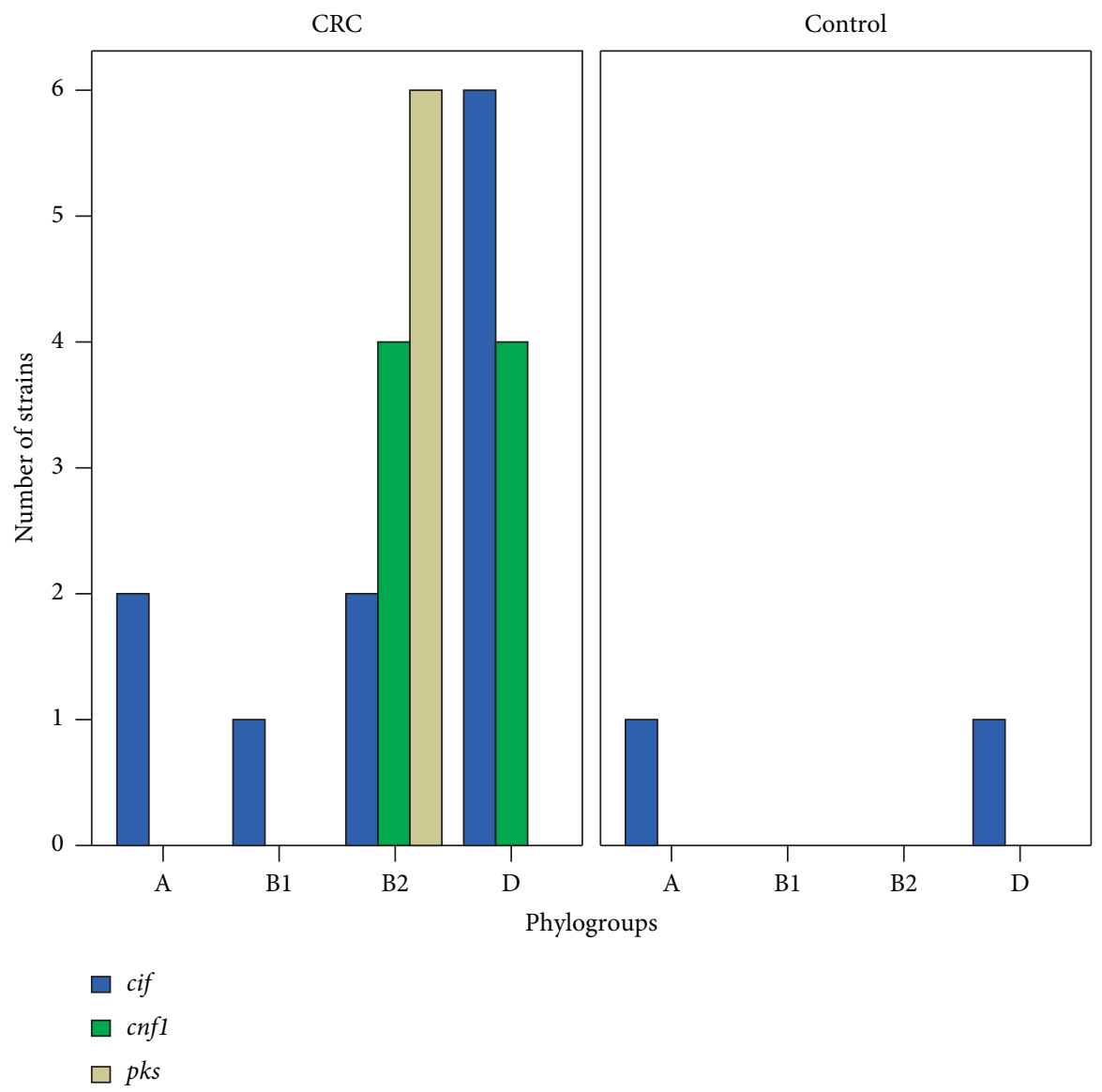

Figure 2: Distribution of cyclomodulin-positive E. coli in various phylogenetic groups.

biofilms in the CRC group $(36.92 \%, n=24 / 65)$ and the control group ( $16.6 \%, n=7 / 42), p=0.03$. Amongst the biofilm-producing strains from the control group, all strains were weak biofilm producers. In contrast, amongst $E$. coli strains of the CRC group, 33.3\% were observed as strong or moderate biofilm producers, while $66.6 \%$ were weak biofilm producers. 
TABLE 2: Phylogroups distribution of $E$. coli isolates according to specimen origins.

\begin{tabular}{lcccccc}
\hline \multirow{2}{*}{ Specimen types } & \multicolumn{4}{c}{ Number (\%) of E. coli strains in various phylogenetic groups } \\
& A & B1 & B2 & D & E & F \\
\hline CRC patients $*$ & $9(13.9)$ & $5(7.7)$ & $24(36.9)$ & $27(41.5)$ & $0(0)$ & $0(0)$ \\
Control group $* *$ & $16(38.1)$ & $8(19)$ & $5(11.9)$ & $7(16.7)$ & $4(9.5)$ & $2(4.7)$ \\
\hline
\end{tabular}

${ }^{*} 65$ E. coli strains were obtained from 50 CRC patients; ${ }^{* *} 42$ E. coli strains were obtained from 50 control group subjects.

TABle 3: Distribution of cyclomodulin-positive E. coli according to phylogenetic groups.

\begin{tabular}{lccccccc}
\hline \multicolumn{7}{c}{ Percentage (number) of E. coli strains from CRC patients in diverse phylogenetic groups } \\
& $\mathrm{A}(n=9)$ & $\mathrm{B} 1(n=5)$ & $\mathrm{B} 2(n=24)$ & $\mathrm{D}(n=27)$ & $\mathrm{E}(n=0)$ & $\mathrm{F}(n=0)$ & All $(n=65)$ \\
\hline cif & $22.2(2)$ & $20(1)$ & $8.3(2)$ & $22.2(6)$ & $0(0)$ & $0(0)$ & $16.9(11)$ \\
cnf & $0(0)$ & $0(0)$ & $16.7(4)$ & $14.8(4)$ & $0(0)$ & $0(0)$ & $12.3(8)$ \\
$p k s$ & $0(0)$ & $0(0)$ & $25(6)$ & $0(0)$ & $0(0)$ & $0(0)$ & $9.2(6)$ \\
$c d t$ & $0(0)$ & $0(0)$ & $0(0)$ & $0(0)$ & $0(0)$ & $0(0)$ & $0(0)$ \\
Cyclomodulin-encoding genes & $22.2(2)$ & $20(1)$ & $50(12) *$ & $37(10) *$ & $0(0)$ & $0(0)$ & $30.7(20) *$
\end{tabular}

\begin{tabular}{lccccccc}
\hline \multicolumn{7}{c}{ Percentage (number) of E. coli strains from the control group in diverse phylogenetic groups } \\
& $\mathrm{A}(n=16)$ & $\mathrm{B} 1(n=8)$ & $\mathrm{B} 2(n=5)$ & $\mathrm{D}(n=7)$ & $\mathrm{E}(n=4)$ & $\mathrm{F}(n=2)$ & All $(n=42)$ \\
\hline cif & $6.2(1)$ & $0(0)$ & $0(0)$ & $14.3(1)$ & $0(0)$ & $0(0)$ & $4.7(2)$ \\
cnf & $0(0)$ & $0(0)$ & $0(0)$ & $0(0)$ & $0(0)$ & $0(0)$ & $0(0)$ \\
pks & $0(0)$ & $0(0)$ & $0(0)$ & $0(0)$ & $0(0)$ & $0(0)$ & $0(0)$ \\
cdt & $0(0)$ & $0(0)$ & $0(0)$ & $0(0)$ & $0(0)$ & $0(0)$ & $0(0)$ \\
Cyclomodulin-encoding genes & $6.2(1)$ & $0(0)$ & $0(0)$ & $14.3(1)$ & $0(0)$ & $0(0)$ & $4.7(2)$ \\
\hline
\end{tabular}

* Some E. coli isolates carried more than one cyclomodulin-encoding genes.

TABle 4: Percentage (number) of biofilm-positive isolates and their biofilm formation ability.

\begin{tabular}{|c|c|c|c|}
\hline & \multirow{2}{*}{ Biofilm-positive strains } & \multicolumn{2}{|c|}{ Biofilm formation category } \\
\hline & & Moderate/strong producers & Weak producers \\
\hline Cyclomodulin-positive strains from CRC patients $(n=20)$ & $60(12)$ & $50(6)$ & $50(6)$ \\
\hline Cyclomodulin-negative strains from CRC patients $(n=45)$ & $26.6(12)$ & $16.6(2)$ & $83.3(10)$ \\
\hline CRC $(n=65)$ & $36.9(24)$ & $33.3(8)$ & $66.6(16)$ \\
\hline Cyclomodulin-positive strains from the control group $(n=2)$ & $50(1)$ & $0(0)$ & $100(1)$ \\
\hline Cyclomodulin-negative strains from the control group $(n=40)$ & $15(6)$ & $0(0)$ & $100(6)$ \\
\hline Control $(n=42)$ & $16.6(7)$ & $0(0)$ & $100(7)$ \\
\hline
\end{tabular}

The number of biofilm-positive strains was significantly higher in cyclomodulin-positive E. coli strains $(60 \%, n=12$ / 2) compared to cyclomodulin-negative E. coli strains (26.6\%, $n=12 / 45), p=0.014$.

\section{Discussion}

CRC is one of the deadliest cancers in the world. Indeed, bacterial infection has long been known as key factor in the etiology of CRC [8]. More recently, accumulating evidence supports that mucosa-associated $E$. coli can affect the development of CRC $[8,29,30]$. However, there are relatively few data available about the prevalence and characterization of mucosa-associated E. coli from CRC specimens.

In our study, the colonic biopsy specimens from CRC and control patients indicated that CRC samples are more colonized by mucosa-associated E. coli strains in comparison to the control group. This result is consistent with the results of other studies [12, 31].

Additionally, EPEC was found to be more prevalent in CRC (23\%) than in control samples in this study $(7.1 \%)$. Magdy et al. have previously reported the presence of EPEC isolates in about $50 \%$ of CRC specimens and $20 \%$ healthy control [16]. Furthermore, recent evidence shows that chronic mucosal EPEC infection could promote molecular pathways involved in CRC carcinogenesis [17]. The higher colonization of biopsy specimens of CRC patients by mucosa-associated $E$. coli can be possibly explained that modifications of the colon mucosal properties in CRC lead to increased expression of adhesion and internalization of pathogens in the tumor microenvironment [8].

In the current study, the distribution of E. coli phylogroups in CRC patient was different from the control group. Most strains from CRC patients belonged to B2 and $\mathrm{D}$ phylogroups, while about $58 \%$ of the strains from the control group belonged to $\mathrm{A}$ and $\mathrm{B} 1$ phylogroups. Thus, it can be concluded that the intestinal mucosa of CRC patients is mainly colonized by $E$. coli strains that are more virulent. Buc et al. [12] and Raisch et al. [11] previously identified higher numbers of E. coli phylogroup B2 in CRC specimens than in controls in two studied. However, no major difference in the abundance of $E$. coli belonging to the phylogenetic group D was found between patients with CRC and diverticulosis in their studies. Group B2 E. coli strains can cause severe infections because their genetic background is favorable for obtaining the high number of virulence factors [32]. Additionally, macrophages are one of the main tumorinfiltrating immune cells which have a pivotal role in cancer 
progression. Interestingly, E. coli of the B2 phylogroup can survive and multiply within macrophages and promote protumoral activities of macrophages independently of colibactin production [33].

In the current research, CRC patients had a substantially higher frequency of cyclomodulin-positive E. coli than the control group, comparable to other studies performed by Bonnet et al. [8], Raisch et al. [11], and Buc et al. [12]. Overall, the findings indicated that cyclomodulin-positive E. coli isolates are implicated in the development of CRC.

We observed cyclomodulin-positive $E$. coli strains in only $4.7 \%$ of the control samples, while Buc et al. [12] and Bonnet et al. [8] observed these isolates in $19.4 \%$ and $26 \%$ of control samples, respectively. The high frequency of cyclomodulin-positive E. coli in their studies compared to ours may be due to they used diverticulosis patients as a control group and dysbiosis of gut microbiota has been reported in diverticulosis patients [34].

In our study, the prevalence of $p k s$-positive E. coli isolated from CRC patients was about $10 \%$, which in contrast to previous research studies is significantly lower. Other research studies have reported $p k s$-positive $E$. coli isolated in CRC patients to range from $26 \%$ to $67 \%[8,11,12,35]$. $p k s+E$. coli isolates are attracting attention because of their ability to induce DNA damage in epithelial cells, leading to genomic instability of mammalian cells [36-40] and thus promote development of colorectal cancer in animal models [35]. Over the course of 30 years, E. coli isolates from fecal samples of populations living in industrialized countries showed a phylogenetic shift from group A toward group B2. Moreover, about $30 \%$ of this commensal B2 E. coli isolates have acquired $p k s$ island by horizontal transfer [41].

Presence of cyclomodulin-encoding gene cif was revealed in $17 \%$ E. coli strains belonging to the CRC patients group. In addition, $12.3 \%$ of $E$. coli strains were positive for cnf gene. More noteworthy, amongst cyclomodulinencoding genes, cif was the most common in our study, in contrast to other research studies whereby $p k s$ has been reported as the most common cyclomodulin-encoding gene $[11,12]$. Thus, our study revealed that in addition to $p k s$, other cyclomodulins especially cif and cnf might play an important role in development of CRC.

In this analysis, the number of biofilm-producing E. coli strains was higher in CRC samples than in control samples $(p<0.05)$. Furthermore, as compared to E. coli strains from healthy mucosa, CRC patients' $E$. coli isolates were high biofilm producers. Interestingly, we also found that cyclomodulin-positive $E$. coli isolates were more likely to generate biofilm than cyclomodulin-negative isolates in the current research. In this regard, Tomkovich et al. have previously reported the presence of mucus-invasive biofilms on the colon mucosa of about half CRC samples and in only $13 \%$ of control specimens [23]. In addition, Dejea et al. for the first time displayed that bacterial biofilms are associated with CRC. Presence of biofilms in tissue may correlate with bacterial invasion and changes in colon tissue biology with increased cellular proliferation [42].

The predominance of adhesin-encoding genes, particularly pili adhesins, that encourage colonization of carcinogenic strains in the intestinal tissue or colorectal tumor microenvironment was not detected in this investigation. It also does not examine into the cyclomodulinproducing strains' carcinogenic potential in animal studies.

As a result, we must examine the frequency and expression of adhesin-encoding genes in E. coli strains isolated from CRC patients and controls in the near future. In addition, we must investigate the ability of cyclomodulinproducing strains to interact with intestinal epithelial cells, as well as their potential carcinogenic effect in animal models.

\section{Conclusion}

Our study showed that E. coli isolates obtained from the CRC patients and controls are a heterogeneous group of isolates with variations in virulence factors, phylogroups, and biofilmforming capacity. To summarize, CRC samples were more colonized by mucosa-associated E. coli, especially cyclomodulin-positive isolates from B2 and D phylogroups with the ability to form biofilms in comparison to the control group. This is the largest study of the prevalence and characterization of mucosa-associated E. coli isolated from CRC patients and a control group in Iran that we are aware of.

\section{Data Availability}

The authors declare the data used to support the findings of this study are available from the corresponding author upon request.

\section{Ethical Approval}

This study was approved by the Research Ethics Committee (IR.TBZMED. REC.1398.409) at Tabriz University of Medical Sciences, Tabriz, Iran.

\section{Disclosure}

This is a report of a database from Ph.D. thesis of the first author registered in the Tabriz University of Medical Sciences.

\section{Conflicts of Interest}

The authors declare that they have no conflicts of interest.

\section{Authors' Contributions}

Roghayeh Nouri, Mohammad Ahangarzadeh Rezaee, and Alka Hasani conceptualized and designed the study. Kourosh Masnadi Shirazi, Bita Sepehri, Simin Sotoodeh, and Afshin Fattahzadeh involved in acquisition of data and sampling. Rohgayeh Nouri, Mohammad Reza Alivand, Babak Abdinia, and Fatemeh Hemmati analyzed and interpreted the study. Roghayeh Nouri and Mohammad Ahangarzadeh Rezaee reviewed the original draft. Mohammad Ahangarzadeh Rezaee and Alka Hasani supervised, reviewed, and edited the study. All authors accepted the final form of the manuscript. 


\section{Acknowledgments}

This study was supported by Infectious and Tropical Diseases Research Center, Tabriz University of Medical Sciences, Tabriz, Iran (62957).

\section{References}

[1] F. Baidoun, K. Elshiwy, and Y. Elkeraie, "Colorectal cancer epidemiology: recent trends and impact on outcomes," Current Drug Targets, vol. 2174, 2020.

[2] P. Favoriti, G. Carbone, M. Greco, F. Pirozzi, R. E. M. Pirozzi, and F. Corcione, "Worldwide burden of colorectal cancer: a review," Updates in Surgery, vol. 68, no. 1, pp. 7-11, 2016.

[3] I. Mármol, C. Sánchez-de-Diego, and A. Pradilla Dieste, "Colorectal carcinoma: a general overview and future perspectives in colorectal cancer," International Journal of Molecular Sciences, vol. 18, no. 1, p. 197, 2017.

[4] M. R. Rubinstein, X. Wang, W. Liu, Y. Hao, G. Cai, and Y. W. Han, "Fusobacterium nucleatum promotes colorectal carcinogenesis by modulating E-cadherin/ $\beta$-catenin signaling via its FadA adhesin," Cell Host \& Microbe, vol. 14, no. 2, pp. 195-206, 2013.

[5] D. Ai, H. Pan, R. Han, X. Li, G. Liu, and L. C. Xia, "Using decision tree aggregation with random forest model to identify gut microbes associated with colorectal cancer," Genes, vol. 10, no. 2, p. 112, 2019.

[6] X. Fan, Y. Jin, G. Chen, X. Ma, and L. Zhang, "Gut microbiota dysbiosis drives the development of colorectal cancer," $D i$ gestion, vol. 102, no. 4, pp. 508-515, 2021.

[7] F. Genua, V. Raghunathan, and M. Jenab, "The role of gut barrier dysfunction and microbiome dysbiosis in colorectal cancer development," Frontiers in Oncology, vol. 11, no. 1016, 2021.

[8] M. Bonnet, E. Buc, P. Sauvanet et al., "Colonization of the human gut by E. coli and colorectal cancer risk," Clinical Cancer Research, vol. 20, no. 4, pp. 859-867, 2014.

[9] F. Haghi, E. Goli, B. Mirzaei, and H. Zeighami, "The association between fecal enterotoxigenic B. fragilis with colorectal cancer," BMC Cancer, vol. 19, no. 1, pp. 879-884, 2019.

[10] J. Gagnière, J. Raisch, J. Veziant et al., "Gut microbiota imbalance and colorectal cancer," World Journal of Gastroenterology, vol. 22, no. 2, pp. 501-18, 2016.

[11] J. Raisch, E. Buc, and M. Bonnet, "Colon cancer-associated B2Escherichia colicolonize gut mucosa and promote cell proliferation," World Journal of Gastroenterology, vol. 20, no. 21, p. 6560, 2014.

[12] E. Buc, D. Dubois, P. Sauvanet et al., "High prevalence of mucosa-associated E. coli producing cyclomodulin and genotoxin in colon cancer," PLoS One, vol. 8, no. 2, p. e56964, 2013.

[13] C. Bernier, P. Gounon, and C. Le Bouguénec, "Identification of an aggregative adhesion fimbria (AAF) type III-encoding operon in enteroaggregative Escherichia coli as a sensitive probe for detecting the AAF-encoding operon family," Infection and Immunity, vol. 70, no. 8, pp. 4302-4311, 2002.

[14] O. Clermont, J. K. Christenson, E. Denamur, and D. M. Gordon, "The ClermontEscherichia coliphylo-typing method revisited: improvement of specificity and detection of new phylo-groups," Environmental Microbiology Reports, vol. 5, no. 1, pp. 58-65, 2013.

[15] D. Kohoutova, D. Smajs, P. Moravkova et al., "Escherichia coli strains of phylogenetic group B2 and D and bacteriocin production are associated with advanced colorectal neoplasia," BMC Infectious Diseases, vol. 14, no. 1, pp. 733-738, 2014.

[16] A. Magdy, M. Elhadidy, M. E. Abd Ellatif et al., "Enteropathogenic Escherichia coli (EPEC): does it have a role in colorectal tumourigenesis? A prospective cohort study," International Journal of Surgery, vol. 18, pp. 169-173, 2015.

[17] O. D. K. Maddocks, A. J. Short, M. S. Donnenberg, S. Bader, and D. J. Harrison, "Attaching and effacing Escherichia coli downregulate DNA mismatch repair protein in vitro and are associated with colorectal adenocarcinomas in humans," PLoS One, vol. 4, no. 5, p. e5517, 2009.

[18] N. Jomehzadeh, K. Ahmadi, and H. Javaherizadeh, "The first evaluation relationship of integron genes and the multidrugresistance in class A ESBLs genes in enteropathogenic Escherichia coli strains isolated from children with diarrhea in Southwestern Iran," Molecular Biology Reports, vol. 2020, pp. 1-7, 2020.

[19] J. Veziant, J. Gagnière, E. Jouberton et al., “Association of colorectal cancer with pathogenicEscherichia coli: focus on mechanisms using optical imaging," World Journal of Clinical Oncology, vol. 7, no. 3, p. 293, 2016.

[20] Y. Yoshikawa, Y. Tsunematsu, and N. Matsuzaki, "Characterization of colibactin-producing Escherichia coli isolated from Japanese patients with colorectal cancer," Japanese Journal of Infectious Diseases, vol. 73, pp. 2020-2066, 2020.

[21] S. Tomkovich and R. Z. Gharaibeh, "Human colon mucosal biofilms and murine host communicate via altered mRNA and microRNA expression during cancer," mSystems, vol. 5, no. 1, pp. e00451-e00419, 2020.

[22] O. Coleman and D. Haller, "Microbe-mucus interface in the pathogenesis of colorectal cancer," Cancers, vol. 13, no. 4, p. 616, 2021.

[23] S. Tomkovich, C. M. Dejea, and K. Winglee, "Human colon mucosal biofilms from healthy or colon cancer hosts are carcinogenic," Journal of Clinical Investigation, vol. 129, no. 4, pp. 1699-1712, 2019.

[24] Y. Cheng, Z. Ling, and L. Li, "The intestinal microbiota and colorectal cancer," Frontiers in Immunology, vol. 11, p. 3100, 2020.

[25] H. M. Martin, B. J. Campbell, and C. A Hart, "Enhanced Escherichia coli adherence and invasion in Crohn's disease and colon cancer," Gastroenterology, vol. 127, no. 1, pp. 80-93, 2004.

[26] J. Versalovic, T. Koeuth, and R. Lupski, "Distribution of repetitive DNA sequences in eubacteria and application to finerpriting of bacterial enomes," Nucleic Acids Research, vol. 9, no. 24, pp. 6823-6831, 1991.

[27] D. Dubois, J. Delmas, and A. Cady, "Cyclomodulins in urosepsis strains of Escherichia coli," Journal of Clinical Microbiology, vol. 48, no. 6, pp. 2122-2129, 2010.

[28] S. Stepanović, D. Vuković, and V. Hola, "Quantification of biofilm in microtiter plates: overview of testing conditions and practical recommendations for assessment of biofilm production by staphylococci," Apmis, vol. 115, no. 8, pp. 891-899, 2007.

[29] L. Salesse, C. Lucas, and M. H. Hoang, "Colibactin-producing Escherichia coli induce the formation of invasive carcinomas in a chronic inflammation-associated mouse model," Cancers, vol. 13, no. 9, p. 2060, 2021.

[30] T. Iyadorai, V. Mariappan, and K. Vellasamy, "Prevalence and association of pks+ Escherichia coli with colorectal cancer in patients at the University Malaya Medical Centre, Malaysia," PLoS One, vol. 15, no. 1, p. e0228217, 2020.

[31] A. Swidsinski, M. Khilkin, and D. Kerjaschki, "Association between intraepithelial Escherichia coli and colorectal cancer," Gastroenterology, vol. 115, no. 2, pp. 281-286, 1998. 
[32] P. Escobar-Páramo, O. Clermont, and A.-B. Blanc-Potard, "A specific genetic background is required for acquisition and expression of virulence factors in Escherichia coli," Molecular Biology and Evolution, vol. 21, no. 6, pp. 1085-1094, 2004.

[33] J. Raisch, N. Rolhion, and A. Dubois, "Intracellular colon cancer-associated Escherichia coli promote protumoral activities of human macrophages by inducing sustained COX-2 expression," Laboratory Investigation, vol. 95, no. 3, pp. 296-307, 2015.

[34] G. Barbara, E. Scaioli, and M. R. Barbaro, "Gut microbiota, metabolome and immune signatures in patients with uncomplicated diverticular disease," Gut, vol. 66, no. 7, pp. 1252-1261, 2017.

[35] J. C. Arthur, E. Perez-Chanona, and M. Mühlbauer, "Intestinal inflammation targets cancer-inducing activity of the microbiota," Science, vol. 338, no. 6103, pp. 120-123, 2012.

[36] H. Zeng, S. Umar, and B. Rust, "Secondary bile acids and short chain fatty acids in the colon: a focus on colonic microbiome, cell proliferation, inflammation, and cancer," International Journal of Molecular Sciences, vol. 20, no. 5, p. 1214, 2019.

[37] T. Secher, A. Samba-Louaka, and E. Oswald, "Escherichia coli producing colibactin triggers premature and transmissible senescence in mammalian cells," PloS One, vol. 8, no. 10, p. e77157, 2013.

[38] A. Iftekhar, H. Berger, and N. Bouznad, "Genomic aberrations after short-term exposure to colibactin-producing E. coli transform primary colon epithelial cells," Nature Communications, vol. 12, no. 1, pp. 1-15, 2021.

[39] P. Dziubańska-Kusibab, H. Berger, and F. Battistini, "Colibactin DNA-damage signature indicates mutational impact in colorectal cancer," Nature Medicine, vol. 26, no. 7, pp. 1063-1069, 2020.

[40] C. Pleguezuelos-Manzano, J. Puschhof, and A. R. Huber, "Mutational signature in colorectal cancer caused by genotoxic pks+ E. coli," Nature, vol. 580, no. 7802, pp. 269-273, 2020.

[41] D. Payros, T. Secher, and M. Boury, "Maternally acquired genotoxic Escherichia coli alters offspring's intestinal homeostasis," Gut Microbes, vol. 5, no. 3, pp. 313-512, 2014.

[42] C. M. Dejea, E. C. Wick, and E. M. Hechenbleikner, "Microbiota organization is a distinct feature of proximal colorectal cancers," Proceedings of the National Academy of Sciences, vol. 111, no. 51, pp. 18321-18326, 2014. 